\title{
Early assessment of the safety and immunogenicity of a third dose (booster) of COVID-19 immunization in Chinese adults
}

\author{
Yuntao Zhang ${ }^{1, *}$, Yunkai Yang ${ }^{1, *}$, Niu Qiao ${ }^{2, *}$, Xuewei Wang ${ }^{1}$, Ling Ding ${ }^{3}$, Xiujuan Zhu ${ }^{3}$, Yu Liang ${ }^{4}$, Zibo Han ${ }^{4}$, \\ Feng Liu ${ }^{2}$, Xinxin Zhang $(\bowtie)^{5}$, Xiaoming Yang $(\varangle)^{1}$ \\ ${ }^{1}$ China National Biotec Group Company Limited, Beijing 100024, China; ${ }^{2}$ Shanghai Institute of Hematology, State Key Laboratory of \\ Medical Genomics, National Research Center for Translational Medicine at Shanghai, Ruijin Hospital Affiliated to Shanghai Jiao Tong \\ University School of Medicine, Shanghai 200025, China; ${ }^{3}$ Beijing Institute of Biological Products, China National Biotec Group Company \\ Limited, Beijing 100176, China; ${ }^{4}$ National Vaccine and Serum Institute, China National Biotec Group Company Limited, Beijing 101111, \\ China; ${ }^{5}$ Department of Infectious Diseases, Research Laboratory of Clinical Virology, Ruijin Hospital Affiliated to Shanghai Jiao Tong \\ University School of Medicine, Shanghai 200025, China
}

(C) Higher Education Press 2022

\begin{abstract}
Inducing durable and effective immunity against severe acute respiratory syndrome coronavirus 2 (SARS-CoV-2) via vaccination is essential to combat the current pandemic of coronavirus disease 2019 (COVID19). It has been noticed that the strength of anti-COVID-19 vaccination-induced immunity fades over time, which calls for an additional vaccination regime, as known as booster immunization, to restore immunity among previously vaccinated populations. Here we report a pilot open-label trial of a third dose of BBIBP-CorV, an inactivated SARS-CoV-2 vaccine (Vero cell), on 136 participants aged between 18 to 63 years. Safety and immunogenicity in terms of neutralizing antibody titers and cytokine/chemokine responses were analyzed as the main endpoint until day 28 . While systemic reactogenicity was either absent or mild, SARS-CoV-2-specific neutralizing antibody titers rapidly arose in all participants within 4 weeks, surpassing the peak antibody titers elicited by the initial two-dose immunization regime. Broad increases of cellular immunity-associated cytokines and chemokines were also detected in the majority of participants after the third vaccination. Furthermore, in an exploratory study, a newly developed recombinant protein vaccine, NVSI-06-08 (CHO Cells), was found to be safe and even more effective than BBIBP-CorV in eliciting humoral immune responses in BBIBP-CorV-primed individuals. Together, these results indicate that a third immunization schedule with either homologous or heterologous vaccine showed favorable safety profiles and restored potent SARS-CoV-2-specific immunity, providing support for further trials of booster vaccination in larger populations.
\end{abstract}

Keywords COVID-19; SARS-CoV-2; vaccine; immunization; booster immunization

\section{Introduction}

As of December 16, 2021, the coronavirus disease 2019 (COVID-19) pandemic has infected over 260 million cases and led to over 5 million deaths since its emergence in late 2019 (data from the World Health Organization (WHO) website). To contain this ongoing pandemic, more than 300 vaccines targeting various elements of SARS-CoV-2 have been developed using a variety of technologies [1],

Received December 9, 2021; accepted December 20, 2021

Correspondence: Xiaoming Yang, yangxiaoming@sinopharm.com; Xinxin Zhang, zhangx@shsmu.edu.cn

*Yuntao Zhang, Yunkai Yang, and Niu Qiao contributed equally to this study. eight of which have been placed on Emergency Use Listing (EUL) by the WHO, including 3 inactivated virus vaccines, 2 mRNA vaccines, and 3 recombinant virus vaccines [2]. However, vaccine-induced immunity typically wanes over time and newly emerged SARS-CoV-2 variants may evade the immunity primed with vaccines developed against older variant(s) [3-6]. To address these concerns, booster immunization using additional dosing schedules has been proposed as a possible solution [7]. Although serological and clinical benefits of booster immunization of several COVID-19 vaccines have been shown in immunocompromised individuals and in countries where additional doses have been made available $[8,9]$, the safety and efficacy of additional vaccination programs warrant thorough assessment in small trials 
before their application in larger populations. Here we report the results of a small open-label trial of booster vaccination on 136 participants who were primed with two doses of BBIBP-CorV, an inactivated vaccine, whose safety and performance have been evaluated by randomized, double-blind, controlled, pilot trials in different age groups [10-12]. Moreover, in an exploratory study, humoral immune responses to a newly developed recombinant vaccine NVSI-06-08 (CHO) were examined in five BBIBP-CorV-primed volunteers. Our study offers support that booster immunization with either homologous or heterologous vaccines is effective in restoring potent immunity against SARS-CoV-2.

\section{Materials and methods}

\section{Patient cohort}

This study was performed with approval by National Vaccine and Serum Institute Ethics Committee. Informed consent was obtained from all enrolled cases. For longitudinal antibody monitoring study, neutralizing antibody titers over the course of 1 year were monitored in 12 individuals after receiving two doses of BBIBP-CorV (Vero) inactivated vaccines. For the open-label trial of homologous booster vaccination, a third dose of BBIBPCorV was administered by intramuscular injection to 136 participants who were primed with two doses of BBIBPCorV. For the exploratory heterologous booster vaccination study, a single dose of NVSI-06-08 recombinant vaccine $(\mathrm{CHO})$ was administered by intramuscular injection to 5 participants who were primed with two doses of BBIBP-CorV. For all participants, the intervals between the 2nd and 3rd vaccinations were more than 6 months. And no participant had reported adverse symptoms post the priming vaccination schedules.

\section{ELISA-based anti-SARS-CoV-2 antibody detection}

The total IgG antibodies against receptor binding domain (RBD) of S-protein and N-protein were detected with antiSARSCoV-2 S-RBD protein Human IgG ELISA Kit (KE30003, ProteinTech, Rosemont, IL, USA) and antiSARS-CoV-2 N protein Human IgG ELISA Kit (KE30001, ProteinTech) according to the manufacturer's instructions. Briefly, serum samples were diluted and added into SARS-CoV-2 S-RBD protein or N-proteincoated plates, followed by incubation with horseradish peroxidase-conjugated anti-human IgG antibodies and then tetramethylbenzidine substrates before adding the stop solution. The plates were read at $450 \mathrm{~nm}$ and $630 \mathrm{~nm}$ using Varioskan ${ }^{\circledR}$ Flash (Thermo Fisher Scientific, Waltham, MA, USA).

\section{Plaque reduction neutralization test}

Plaque reduction neutralization test was used to measure the neutralization capacity induced by the vaccine against live SARS-CoV-2 viruses in a Biosafety Level 3 laboratory as described earlier [12]. Briefly, in each experiment, $0.3 \mathrm{~mL}$ of 4 -fold serial dilutions were added to $0.2 \mathrm{~mL}$ of $\sim 600 \mathrm{pfu} / \mathrm{mL}$ of SARS-CoV-2 and incubated for $1 \mathrm{~h}$ at $37^{\circ} \mathrm{C}$. The $0.5 \mathrm{~mL}$ mixtures were then added to a monolayer of Vero cells in six-well plates and incubated for $1 \mathrm{~h}$ at $37^{\circ} \mathrm{C}$. After removing the supernatant, $1.0 \mathrm{~mL}$ of $0.9 \%(\mathrm{w} / \mathrm{v})$ LMP agarose (Promega) in $2 \times$ DMEM supplemented with $4 \%(\mathrm{v} / \mathrm{v})$ FBS was added onto the infected cells. After further incubation for 2 days at $37^{\circ} \mathrm{C}$ in a $5 \% \mathrm{CO}_{2}$ atmosphere, the wells were stained with $0.05 \%(\mathrm{w} / \mathrm{v})$ crystal violet dissolved in $8 \%(\mathrm{v} / \mathrm{v})$ formaldehyde to visualize the plaques. IC50 values were determined using the Reed-Mench method.

\section{Pseudovirus-based SARS-CoV-2 neutralizing antibody detection}

Pseudovirus-based neutralizing antibody detection was as previously described [12]. The collected plasma was first heated at $56{ }^{\circ} \mathrm{C}$ to inactivate the complement proteins, and then diluted with culture media and incubated with pseudovirus for one hour at $37{ }^{\circ} \mathrm{C}$. Subsequently, the virus-plasma mix was added into Huh7 cells for $24 \mathrm{~h}$ before measuring luciferase activity using the EnSight@ Multimode Microplate Reader (HH34000000, PerkinElmer, Singapore). The $50 \%$ neutralization titer $\left(\mathrm{NT}_{50}\right)$ was used to evaluate the neutralizing ability of the antibodies in the plasma.

\section{Clinical laboratory tests}

Routine blood tests - including analysis of blood cell counts and blood cell percentages - were performed with the Sysmex XN-1000 Hematology Analyzer. Coagulationrelated markers were detected with the STA-R MAX coagulation analyzer. Subsets of lymphocytes were labeled with the BD Multitest ${ }^{\mathrm{TM}} 6$-color TBNK and detected by the BD FACSCanto ${ }^{\mathrm{TM}}$. Biochemical analyses, including albumin, alkaline phosphatase, creatine kinase, creatinine, lactate dehydrogenase, and total protein, were performed by ACCELERATOR a3600/ARCHITECT c16000 Automatic biochemistry analyzer (Abbott Diagnostics).

\section{Cytokine and chemokine measurements}

Plasma concentrations of 48 cytokines/chemokines were measured in duplicate using the Bio-Plex Pro Human Cytokine Screening 48-Plex Panel according to the manufacturer's instructions (Bio-Rad, Cat\#: 12007283). 
In total, 141 samples from the booster immunization cohort, 3 healthy volunteers (negative control), and 4 acute COVID-19 patients (positive control) were tested. Test results were analyzed according to Liu et al.'s study [3].

\section{Statistical analysis}

Data are presented as the geometric mean titers. Standard deviation (SD) is estimated on $\log _{10}$ transformed neutralization titers. Categorical variables were compared using Pearson's Chi-square or Fisher's exact test. Unpaired twosided Student's $t$-tests were used to compare two unpaired groups of variables. Paired two-sided Student's $t$-tests were used to compare the significance of paired samples. Wilcoxon tests were performed to compare non-normally distributed continuous variables. Statistical significance was defined as $P<0.05$ and the R version 4.1 .0 was used for all statistical analysis. The results were visualized by the ggplot2 (version 3.3.3), ComplexHeatmap (version 2.8.0), and supraHex (version 1.30.0) R packages [13-15].

\section{Results}

\section{Booster vaccination of BBIBP-CorV enhances humoral immunity against SARS-CoV-2}

To determine the durability of BBIBP-CorV-induced immunity, we monitored, for up to a year, the titers of neutralizing antibodies (nAbs) in serum samples of 12 volunteers, who were previously immunized with two doses of BBIBP-CorV (Table S1). Blood samples were collected before and at six time points after the first dose (day $14 / 21$, and month 3/6/9/12). Clinical blood laboratory tests showed that the vaccine was well tolerated in all 12 participants (Table S2). As expected, nAb titers showed a consistent increase within the first 3 months, with an almost 5-fold increase during this time (geometric mean titers (GMT): 18.0 at day 14 vs. 91.1 at month 3; Fig. 1A and 1B). However, starting from month 6 , the nAb titers regressed to low levels similar to those of at day 14 post the first vaccination (Fig. 1A and 1B).

Next, to assess if $n A b$ titers could be restored by booster immunization, we analyzed the nAb titers of 136 individuals, who participated in a small open-label trial to administer a third dose of BBIBP-CorV (Table 1 and Table S3). This cohort was previously primed with two doses of BBIBP-CorV (21-28 days apart), and 6-16 months had passed since the first vaccination. We confirmed that the sera collected at the onset of this study contained only baseline anti-SARS-CoV-2 nAbs titers (nAb GMT: 18.3) (Fig. 1C and 1D). In contrast, after the third dose, nAbs titers rapidly increased in nearly all subjects within the first month, from 18.3 at day 3 to 439.8 at day 28 (24-fold increase, GMT; Fig. 1C and 1D). The rate of $\mathrm{nAb}$ increase appeared to be relatively constant during the first 14 days and then declined between day 14 and day 28 , suggesting that the $\mathrm{nAb}$ titers might reach a plateau level in about a month. Interestingly, nAb titers at day 28 post the third dose (GMT: 439.8) were even higher than those at month 3 after the first dose (GMT: 91.1), a 4.83-fold increase, indicating that booster vaccination induced a stronger humoral response than the initial twodose immunization regime. Furthermore, although the sera of female participants contained a slightly higher $\mathrm{nAb}$ titer at day 3 (female 27.15 vs. male 19.61, GMT, $P=0.002$ ), this difference was not evident at other times (Table 1). Last, systemic reactogenicity in this cohort was either absent or mild after the third immunization, with only minor side effects such as local granuloma formation and temporary fatigue observed in three participants.

\section{Booster vaccination of BBIBP-CorV activates cytokine and chemokine responses}

To evaluate cellular immune responses to the third dose of BBIBP-CorV, we examined the levels of 48 cytokines/ chemokines in the plasma of the above sequentially vaccinated individuals (Table S4). For comparison, plasma samples of four acutely SARS-CoV-2-infected individuals were used as positive controls, and those of three healthy individuals prior to being vaccinated were used as negative controls. As shown in Fig. 2A, after the third dose of BBIBP-CorV, there was a broad low-to-moderate increase of cytokine and chemokine levels in the majority of sequentially vaccinated samples (Fig. 2 and Fig. S1). The cytokine and chemokine responses were already evident at day 3 , and the temporal patterns of many were coordinated over time in the next 25 days (Fig. 2B and Fig. S2). Nevertheless, the levels of most cytokines and chemokines subsided to low levels by day 28 (Fig. 2B). These results were in congruence with the expectation that the additional vaccination activated broad cellular immune responses in the hosts.

On the other hand, in consistence with previous studies, a number of pro-inflammatory cytokines were prominently induced in acutely infected samples (positive controls) (Figs. 2B, 2C, and S1), including IL-6, IL-18, CXCL10, and IL-1RN whose increases were implicated in cytokine storm syndromes in severe COVID-19 cases [16]. It is worth noting that even though these pro-inflammatory cytokines were also significantly elevated in sequentially vaccinated samples, their levels in the latter were much lower than in acute COVID-19 samples (Fig. 2C), suggesting that pathogenic pro-inflammatory responses were not activated in vaccinated groups, a conclusion that was in agreement with the overall lack of adverse symptoms in the individuals post booster immunization. 

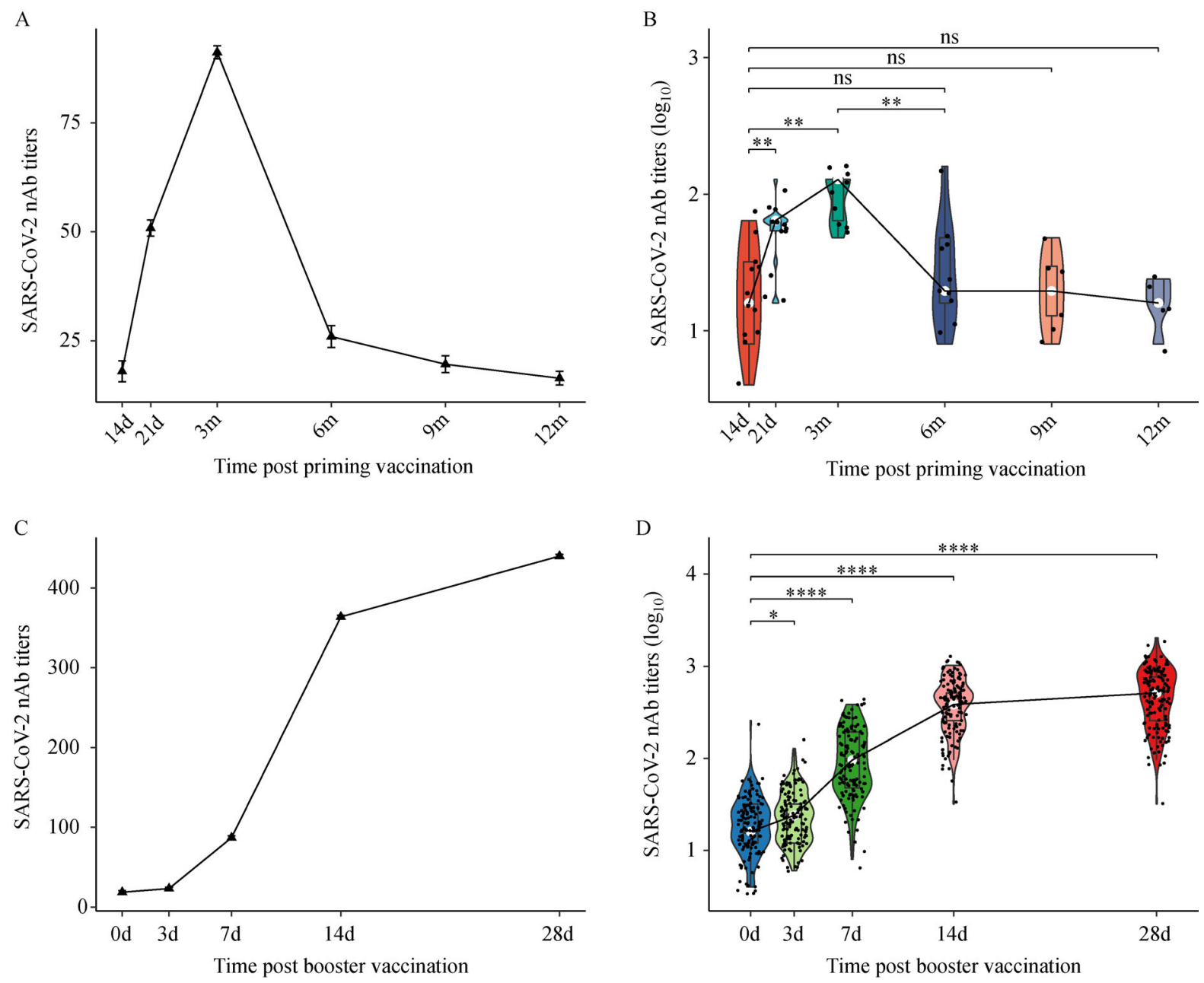

Fig. 1 Booster vaccination of BBIBP-CorV enhances humoral immunity against SARS-CoV-2. (A,B) Neutralizing antibody titers of 12 individuals during the first year after receiving two doses of BBIBP-CorV vaccine. Geometric mean titer (GMT) values (A) and $\log _{10}$ values of neutralizing antibodies (B) are shown. (C,D) Neutralizing antibody titers of 136 BBIBP-CorV-primed individuals during the first month after receiving a third dose of BBIBP-CorV vaccine. GMT values (A) and $\log _{10}$ values of neutralizing antibodies (B) are shown. Standard deviation (SD) is estimated on $\log _{10}$ transformed neutralization titers. ${ }^{*} P_{\text {adj }}<0.05$, ${ }^{* *} P_{\text {adj }}<0.01, * * * * P_{\text {adj }}<0.0001$; ns, not significant; d, day; m, month.

Table 1 General information about 136 cases enrolled in the booster vaccination

\begin{tabular}{|c|c|c|c|}
\hline Characteristics & Female $(n=64)$ & Male $(n=72)$ & $P$ value \\
\hline Age (year) & & & 0.372 \\
\hline$<40$ & $43(67.2)$ & $40(55.6)$ & \\
\hline $40-60$ & $20(31.2)$ & $30(41.7)$ & \\
\hline$\geqslant 60$ & $1(1.6)$ & $2(2.8)$ & \\
\hline Post-0 day & $18.48(0.33)$ & $18.17(0.28)$ & 0.889 \\
\hline Post-3 days & $27.15(0.27)$ & $19.61(0.26)$ & 0.002 \\
\hline Post-7 days & $95.35(0.38)$ & $79.85(0.35)$ & 0.222 \\
\hline Post-14 days & $384.15(0.28)$ & $346.85(0.32)$ & 0.393 \\
\hline Post-28 days & $453.11(0.31)$ & $428.36(0.33)$ & 0.661 \\
\hline
\end{tabular}

Data are geometric mean titers (SD) or $n(\%)$. SD is estimated on $\log _{10}$ transformed neutralization titers. Fisher exact test was performed to compare categorical variables, and Wilcoxon tests were performed to compare continuous variables between groups.

\section{Heterologous vaccination with recombinant vaccine NVSI-06-08 enhances immunity in BBIBP-CorV- primed individuals}

In an exploratory study, we also evaluated the safety and efficacy of heterologous vaccination schedule, by vaccinating five BBIBP-CorV-primed volunteers with a single dose of NVSI-06-08, a recombinant COVID-19 vaccine (CHO Cells) composed of a heterologous trimer of receptor binding domain (RBD) of SARS-CoV-2 (Table 2). The interval between the initial and booster vaccination schedules was also more than six months. Blood samples were collected between day 14 and day 28 post the third vaccination to test neutralizing antibodies titers. For comparison, samples from five individuals receiving homologous vaccinations (BBIBP-CorV was 

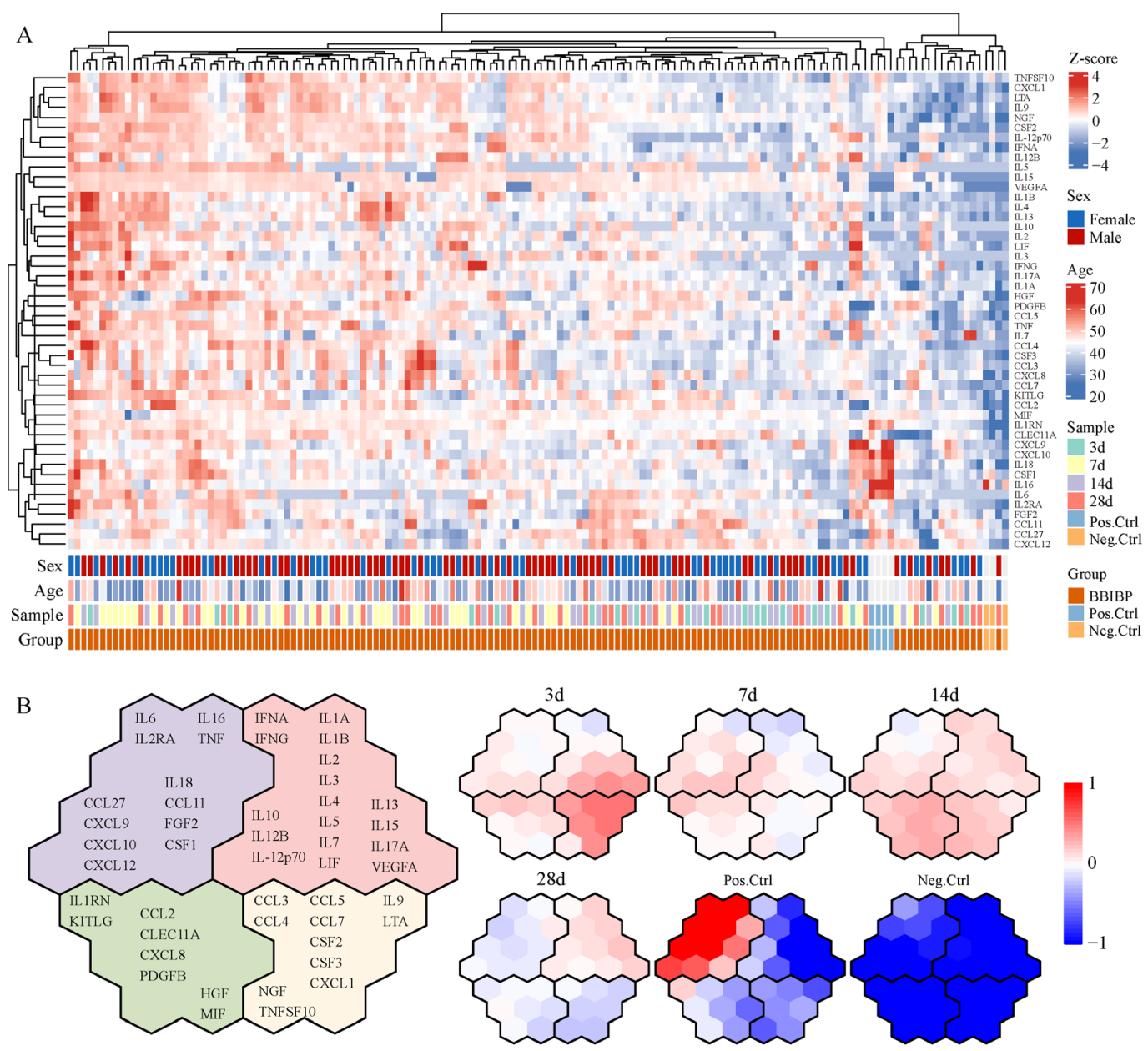

C
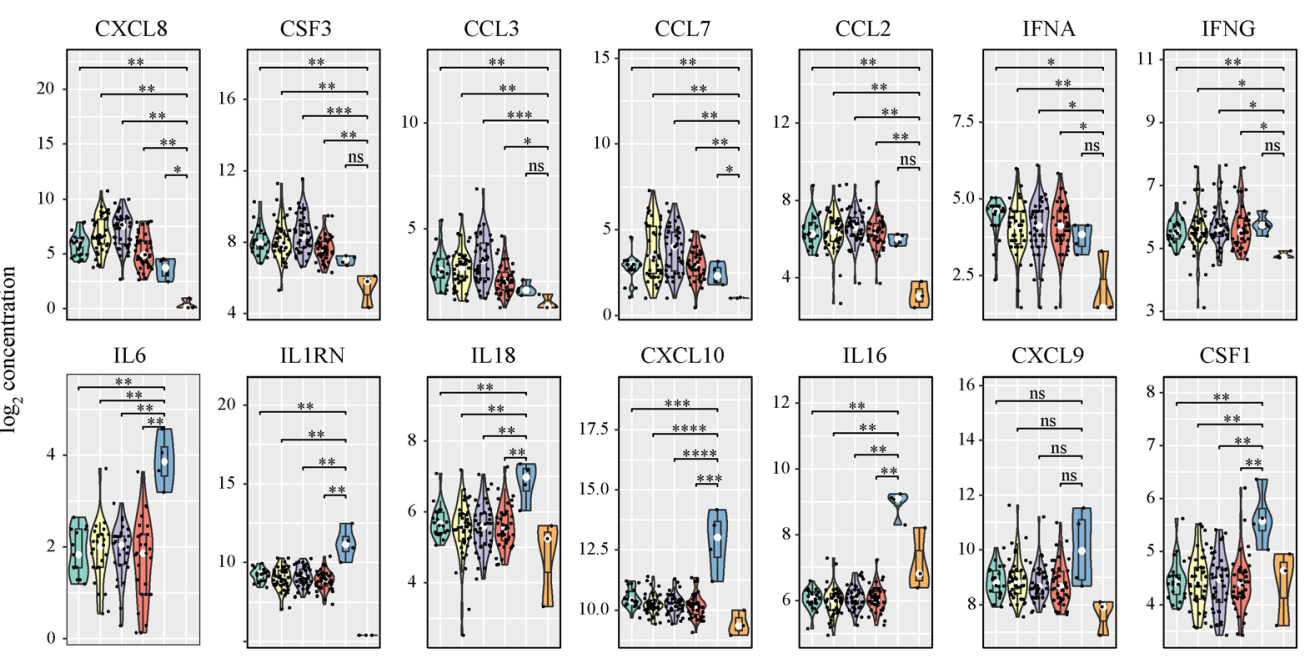

CXCL10
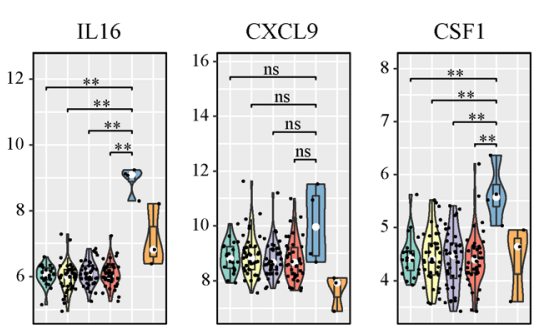

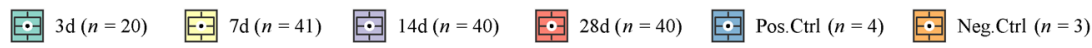

Fig. 2 Booster vaccination of BBIBP-CorV activates cytokine and chemokine responses. (A) Heat map of the measurement of 48 cytokines/ chemokines. Colors indicate Z-scores. Factor names are shown at right. SARS-CoV-2 infection-related factors were labeled in red. Information of the age and sex of the subjects, and sample collection time are shown on the right. BBIBP, samples from subjects receiving the third dose of BBIBP-CorV vaccination; Pos.Ctrl, positive control (acutely infected COVID-19 cases); Neg.Ctrl, negative control. (B) Self-organizing map of four clusters of cytokines and chemokines in plasma samples of subjects receiving a third dose of BBIBP-CorV. The samples were divided according to the time of blood collection. Red represents higher levels and blue represents lower levels. (C) Violin plots of representative cytokines/chemokines induced by the third dose of BBIBP-CorV. $P$ values of Wilcoxon tests are shown on each plot. ${ }^{*} P_{\text {adj }}<0.05,{ }^{* *} P_{\text {adj }}<0.01,{ }^{* * *} P_{\text {adj }}<0.001,{ }^{* * * *} P_{\text {adj }}<0.0001$; ns, not significant; d, day. 
Table 2 Characteristics of individuals in the exploratory study using a third dose of BBIBP-CorV vaccine and NVSI-06-08 vaccine

\begin{tabular}{|c|c|c|c|c|c|c|c|}
\hline \multirow{2}{*}{ Type } & \multirow{2}{*}{ Age } & \multirow{2}{*}{ Sex } & \multirow{2}{*}{$\begin{array}{l}\text { Time interval to the } 2 \text { nd dose } \\
\text { (month) }\end{array}$} & \multicolumn{2}{|c|}{ Before 3rd vaccination } & \multicolumn{2}{|c|}{ After 3rd vaccination (14-28 days) } \\
\hline & & & & $\mathrm{nAb}$ titers & Sampling date & $\mathrm{nAb}$ titers & Sampling date \\
\hline $\mathrm{B}, \mathrm{B}, \mathrm{B}$ & 57 & Male & 6 & 24 & $2021 / 8 / 5$ & 512 & $2021 / 8 / 19$ \\
\hline $\mathrm{B}, \mathrm{B}, \mathrm{B}$ & 61 & Female & 6 & 24 & $2021 / 8 / 5$ & 384 & $2021 / 9 / 2$ \\
\hline $\mathrm{B}, \mathrm{B}, \mathrm{B}$ & 50 & Male & 13 & 12 & $2021 / 8 / 9$ & 256 & $2021 / 8 / 23$ \\
\hline $\mathrm{B}, \mathrm{B}, \mathrm{B}$ & 44 & Female & 14 & 12 & $2021 / 7 / 30$ & 256 & $2021 / 8 / 26$ \\
\hline $\mathrm{B}, \mathrm{B}, \mathrm{B}$ & 62 & Male & 6 & 16 & $2021 / 8 / 9$ & 384 & $2021 / 9 / 6$ \\
\hline $\mathrm{B}, \mathrm{B}, \mathrm{N}$ & 40 & Female & 6 & / & l & 1280 & 2021/8/19 \\
\hline $\mathrm{B}, \mathrm{B}, \mathrm{N}$ & 27 & Male & 6 & I & l & 960 & $2021 / 9 / 2$ \\
\hline $\mathrm{B}, \mathrm{B}, \mathrm{N}$ & 27 & Male & 6 & I & I & 1280 & $2021 / 8 / 23$ \\
\hline $\mathrm{B}, \mathrm{B}, \mathrm{N}$ & 31 & Male & 6 & 5 & $2021 / 7 / 30$ & 1240 & $2021 / 8 / 26$ \\
\hline $\mathrm{B}, \mathrm{B}, \mathrm{N}$ & 30 & Male & 9 & 5 & $2021 / 8 / 9$ & 960 & $2021 / 9 / 6$ \\
\hline
\end{tabular}

$\mathrm{nAb}$, neutralizing antibodies; $\mathrm{B}, \mathrm{B}, \mathrm{B}, \mathrm{BBIBP}-\mathrm{CorV}$-primed individuals with a third dose of BBIBP-CorV vaccine; B,B,N, BBIBP-CorV-primed individuals with a third dose of NVSI-06-08 vaccine.

used for both priming and booster vaccination) at about the same time were used. As shown in Fig. 3, the geometric mean nAbs titers in the homologous-vaccination group were 345.8 (GMT), whereas their counterparts in the heterologous-vaccination group were 1133.6 (GMT), a 3.28 -fold difference, suggesting that the recombinant vaccine NVSI-06-08 might have induced stronger humor immunity than the BBIBP-CorV in individuals primed with the latter (Fig. 3A). Moreover, we observed no statistical significances in the neutralizing capacity induced by NVSI-06-08 against multiple live or pseudotyped SARS-CoV-2 variants (i.e., Alpha, Beta, Gamma, Delta, and Kappa) (Fig. 3B and Fig. 3C).

\section{Discussion}

Here we have examined the safety and immunogenicity of booster anti-SARS-CoV-2 immunization in a small group of Chinese adult volunteers (over 18 years of age), who were previously immunized with two doses of the inactivated vaccine BBIBP-CorV for over 6 months and whose neutralization antibody titers (GMT) had reduced to baseline levels. In one study, we analyzed the humoral responses of 136 participants to a third dose of BBIBPCorV. This homologous vaccination schedule quickly restores neutralizing antibody titers (GMT) to levels that are even higher than the peak antibody titers generated by the priming two doses. Additionally, low-to-moderate induction of a panel of 48 cytokines/chemokines were observed in the plasma of most participants, which was indicative of broad cellular immune responses to the additional vaccination. Taking together with the observation that no significant adverse effects were identified in this cohort, our study suggests that booster immunization of BBIBP-CorV is safe and effective in restoring antiCOVID-19 immunity among individuals who were previously immunized by the same vaccine. In another study, five individuals participated in an exploratory study of boosting immunization by a third dose of a heterologous, recombinant vaccine NVSI-06-08. The blood samples from this heterologous booster vaccination group also showed a rapid rise of nAbs titers, reaching levels that were about three times more than those in the homologous vaccination group.

Of note, while this manuscript was in preparation, several studies were published, which examined the reactogenicity and immunogenicity of inactivated vaccine-primed individuals (CoronaVac or BBIBP-CorV) after receiving a third dose of a homologous vaccine or a heterologous vaccine (ZF2001, an RBD dimer subunit recombinant vaccine) [17-19]. In these studies, a third dose elicited enhanced humoral immunity against multiple SARS-CoV-2 variants, including the highly infectious Delta variant [17-19]. Though we used a different priming (BBIBP-CorV vs. CoronaVac) or booster (NVSI-06-08 vs. ZF2001) vaccine, our study support that both homologous and heterologous vaccinations could boost the immunity of individuals previously vaccinated by inactivated virus vaccines. Additionally, enhanced immunity was observed after a third dose of BNT162b2 (a mRNA vaccine) as homologous vaccination [20,21], or as heterologous vaccination for ChAdOx1-nCoV-19-primed individuals [22]. A more recent study even tested seven different COVID-19 vaccines as a third dose after two doses of ChAdOx1 nCov-19 or BNT162b2 [23]. Together, these studies provided multiple lines of independent evidence that immunity could be enhanced by booster immunization with different types of vaccines. Because different vaccines might produce different antigens, various combinations of heterologous immunization schedules could be a means to increase the diversity of B or T cell immunity and prolong protection of the already large number of SARS-CoV-2 variants. 

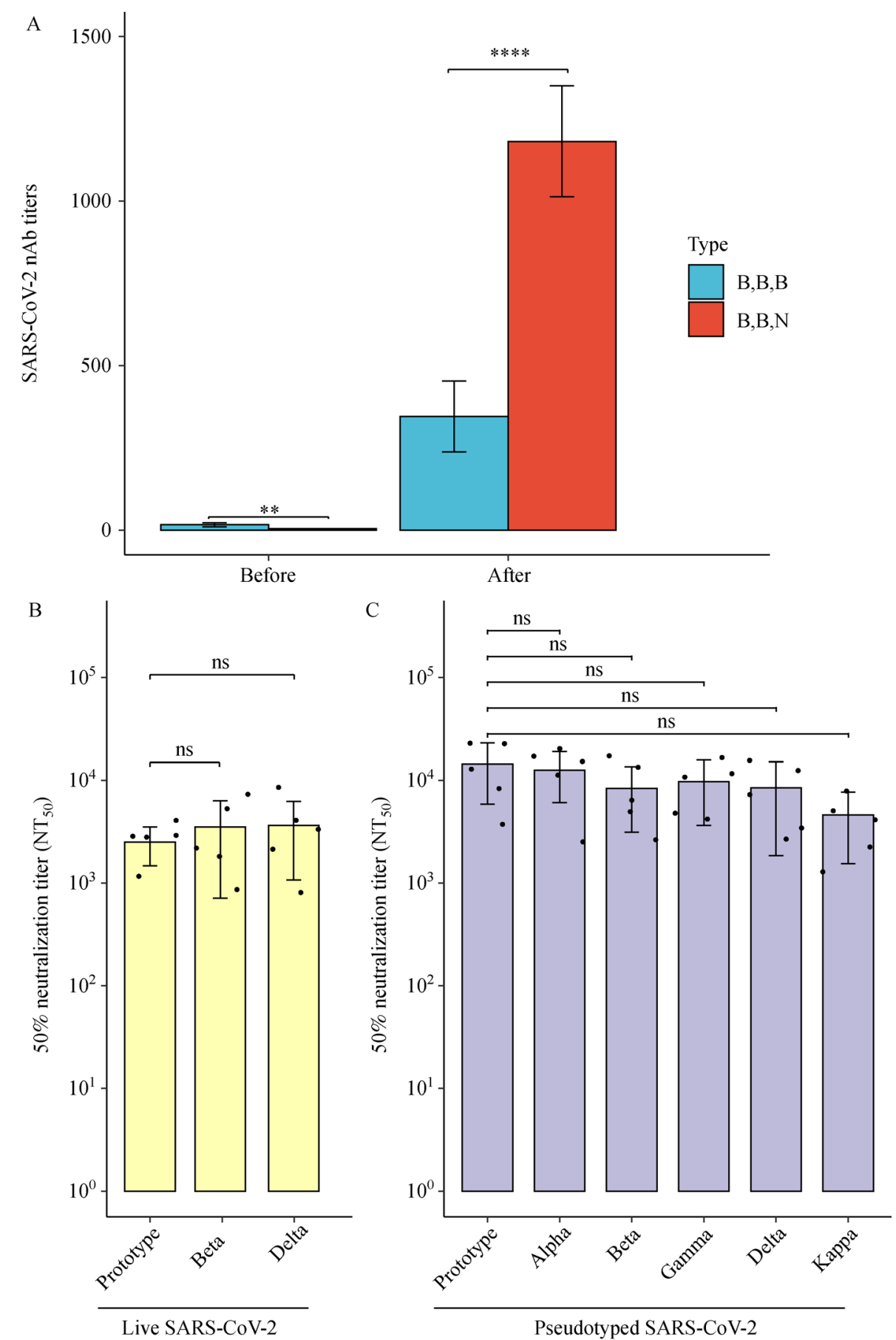

Fig. 3 Heterologous vaccination with recombinant vaccine NVSI-06-08 enhances immunity in BBIBP-CorV-primed individuals. (A) Neutralizing antibody titers of BBIBP-CorV-primed individuals at 14-28 days after receiving a third dose of BBIBP-CorV vaccine $(\mathrm{B}, \mathrm{B}, \mathrm{B} ; n=5)$ or NVSI-06-08 vaccine (B,B,N; $n=5)$. Geometric mean titer (GMT) values are shown and standard deviation (SD) is calculated based on the raw neutralization titers. (B) $\mathrm{NT}_{50}$ of plaque reduction neutralization test (PRNT) was used to evaluate the neutralization capacity induced by the vaccine against live SARS-CoV-2 variants. (C) $\mathrm{NT}_{50}$ of pseudovirus-based neutralizing antibody detection tests against a panel of pseudotyped SARS-CoV-2 variants. $* * P<0.01, * * * * P<0.0001$; ns, not significant.

In summary, recent studies, including our study here, suggest that booster vaccination, especially heterologous vaccination, could offer potent protection against SARSCoV-2 among previously vaccinated populations. How- ever, thus far, all studies assessing the safety and immunogenicity of a third vaccination schedule were based on relatively small open-label trials, nor did they include young children or adults older than 65 years of 
age. Therefore, the reactogenicity and immunogenicity of additional vaccination schedules shall be further interrogated by multi-center studies with larger sample sizes. Furthermore, all recent studies on the efficacy of additional dosing schedules only examined humoral immune responses during the first month after the third dose, and questions remain regarding how long they could last. Last, even though $\mathrm{T}$ lymphocytes play a critical role for vaccine-induced immunity, few studies have yet explicitly evaluated whether T cell immunity also becomes faded as quickly as humoral immune responses, or whether they can also be enhanced by additional dosing schedules. Future studies on these issues shall prove invaluable for developing effective immunity against emerging infectious viruses such as SARS-CoV-2.

\section{Acknowledgements}

This work was supported by the National Program on Key Research Project of China (Nos. 2020YFA0707500, 2016YFD0500301, 2017YFC0840300, and 2020YFC0842100), National Mega projects of China for Major Infectious Diseases (No. 2016ZX10004001003), National Mega Projects of China for New Drug Creation (No. 2018ZX09734-004), Beijing Science and Technology Plan (No. Z201100005420014). The National Vaccine and Serum Institute, China National Biotec Group Company Limited; Beijing Institute of Biological Products, China National Biotec Group Company Limited; and China National Biotec Group Company Limited provided the study product and oversaw all trial operations.

\section{Compliance with ethics guidelines}

Yuntao Zhang, Yunkai Yang, Xuewei Wang, and Xiaoming Yang are employees of China National Biotec Group Company Limited, Ling Ding and Xiujuan Zhu are employees of Beijing Institute of Biological Products, China National Biotec Group Company Limited, and Yu Liang and Zibo Han are employees of National Vaccine and Serum Institute, China National Biotec Group Company Limited which developed the vaccine and funded the trial. All other authors declare no competing interests. All procedures followed were in accordance with the ethical standards of the responsible committee on human experimentation (institutional and national) and with the Helsinki Declaration of 1975, as revised in 2000 (5). Informed consent was obtained from all patients for being included in the study.

Electronic Supplementary Material Supplementary material is available in the online version of this article at https://oi.org/ $10.1007 / \mathrm{s} 11684-021-0914-\mathrm{x}$ and is accessible for authorized users.

\section{References}

1. Dai L, Gao GF. Viral targets for vaccines against COVID-19. Nat
Rev Immunol 2021; 21(2): 73-82

2. WHO. Considerations for the Assessment of COVID-19 Vaccines for Listing by WHO. 2020. https://www.who.int/publications/m/ item/considerations-for-the-assessment-of-covid-19-vaccines-forlisting-by-who (accessed December 22, 2021)

3. Liu Y, Zhang C, Huang F, Yang Y, Wang F, Yuan J, Zhang Z, Qin Y, Li X, Zhao D, Li S, Tan S, Wang Z, Li J, Shen C, Li J, Peng L, Wu W, Cao M, Xing L, Xu Z, Chen L, Zhou C, Liu WJ, Liu L, Jiang C. Elevated plasma levels of selective cytokines in COVID-19 patients reflect viral load and lung injury. Natl Sci Rev 2020; 7(6): 1003-1011

4. Mallapaty S. COVID vaccines slash viral spread - but Delta is an unknown. Nature 2021; 596(7870): 17-18

5. Safari I, Elahi E. Evolution of the SARS-CoV-2 genome and emergence of variants of concern. Arch Virol 2021; [Epub ahead of print] doi: 10.1007/s00705-021-05295-5

6. Shrotri M, Navaratnam AMD, Nguyen V, Byrne T, Geismar C, Fragaszy E, Beale S, Fong WLE, Patel P, Kovar J, Hayward AC, Aldridge RW; Virus Watch Collaborative. Spike-antibody waning after second dose of BNT162b2 or ChAdOx1. Lancet 2021; 398 (10298): 385-387

7. Shekhar R, Garg I, Pal S, Kottewar S, Sheikh AB. COVID-19 vaccine booster: to boost or not to boost. Infect Dis Rep 2021; 13(4): 924-929

8. Bagacean C, Letestu R, Al-Nawakil C, Brichler S, Lévy V, Sritharan N, Delmer AJ, Dartigeas C, Leblond V, Roos-Weil D, Tomowiak C, Merabet F, Béné MC, Clavert A, Chaoui D, Genet P, Guieze R, Laribi K, Drénou B, Willems L, Puppinck C, Legendre H, Troussard X, Malartre S, Cymbalista F, Michallet AS. Humoral response to mRNA anti-COVID-19 vaccines BNT162b2 and mRNA-1273 in patients with chronic lymphocytic leukemia. Blood Adv 2021; [Epub ahead of print] doi: 10.1182/bloodadvances.2021006215

9. Hall VG, Ferreira VH, Ku T, Ierullo M, Majchrzak-Kita B, Chaparro C, Selzner N, Schiff J, McDonald M, Tomlinson G, Kulasingam V, Kumar D, Humar A. Randomized trial of a third dose of mRNA1273 vaccine in transplant recipients. N Engl J Med 2021; 385(13): 1244-1246

10. Guo W, Duan K, Zhang Y, Yuan Z, Zhang YB, Wang Z, Zhao D, Zhang H, Xie Z, Li X, Peng C, Zhang W, Yang Y, Chen W, Gao X, You W, Wang XW, Shi Z, Wang Y, Yang XQ, Zhang L, Huang L, Wang Q, Lu J, Yang YL, Guo J, Zhou W, Wan X, Wu C, Wang W, Du J, Nian X, Li XH, Huang S, Shen S, Xia S, Pan A, Yang X. Safety and immunogenicity of an inactivated SARS-CoV-2 vaccine in healthy adults aged 18 years or older: a randomized, double-blind, placebo-controlled, phase 1/2 trial. EClinicalMedicine 2021; 38: 101010

11. Xia S, Duan K, Zhang Y, Zhao D, Zhang H, Xie Z, Li X, Peng C, Zhang Y, Zhang W, Yang Y, Chen W, Gao X, You W, Wang X, Wang Z, Shi Z, Wang Y, Yang X, Zhang L, Huang L, Wang Q, Lu J, Yang Y, Guo J, Zhou W, Wan X, Wu C, Wang W, Huang S, Du J, Meng Z, Pan A, Yuan Z, Shen S, Guo W, Yang X. Effect of an inactivated vaccine against SARS-CoV-2 on safety and immunogenicity outcomes: interim analysis of 2 randomized clinical trials. JAMA 2020; 324(10): 951-960

12. Xia S, Zhang Y, Wang Y, Wang H, Yang Y, Gao GF, Tan W, Wu G, Xu M, Lou Z, Huang W, Xu W, Huang B, Wang W, Zhang W, Li N, Xie Z, Zhu X, Ding L, You W, Zhao Y, Zhao J, Huang L, Shi X, 
Yang Y, Xu G, Wang W, Liu P, Ma M, Qiao Y, Zhao S, Chai J, Li Q, $\mathrm{Fu} \mathrm{H}, \mathrm{Xu} \mathrm{Y,} \mathrm{Zheng} \mathrm{X,} \mathrm{Guo} \mathrm{W,} \mathrm{Yang} \mathrm{X.} \mathrm{Safety} \mathrm{and} \mathrm{immunogenicity}$ of an inactivated COVID-19 vaccine, BBIBP-CorV, in people younger than 18 years: a randomised, double-blind, controlled, phase 1/2 trial. Lancet Infect Dis 2021; [Epub ahead of print] doi: 10.1016/S1473-3099(21)00462-X

13. Gu Z, Eils R, Schlesner M. Complex heatmaps reveal patterns and correlations in multidimensional genomic data. Bioinformatics 2016; 32(18): 2847-2849

14. Fang H, Gough J. supraHex: an R/Bioconductor package for tabular omics data analysis using a supra-hexagonal map. Biochem Biophys Res Commun 2014; 443(1): 285-289

15. Tan Y, Jiang L, Wang K, Fang H. I3: a self-organising learning workflow for intuitive integrative interpretation of complex genetic data. Genomics Proteomics Bioinformatics 2019; 17(5): 503-510

16. Tan Y, Zhang W, Zhu Z, Qiao N, Ling Y, Guo M, Yin T, Fang H, Xu X, Lu G, Zhang P, Yang S, Fu Z, Liang D, Xie Y, Zhang R, Jiang L, Yu S, Lu J, Jiang F, Chen J, Xiao C, Wang S, Chen S, Bian XW, $\mathrm{Lu} \mathrm{H}$, Liu F, Chen S. Integrating longitudinal clinical laboratory tests with targeted proteomic and transcriptomic analyses reveal the landscape of host responses in COVID-19. Cell Discov 2021; 7(1): 42

17. Ai J, Zhang H, Zhang Q, Zhang Y, Lin K, Fu Z, Song J, Zhao Y, Fan M, Wang H, Qiu C, Zhou Y, Zhang W. Recombinant protein subunit vaccine booster following two-dose inactivated vaccines dramatically enhanced anti-RBD responses and neutralizing titers against SARS-CoV-2 and Variants of Concern. Cell Res 2021; [Epub ahead of print] doi: 10.1038/s41422-021-00590-x

18. Cao Y, Hao X, Wang X, Wu Q, Song R, Zhao D, Song W, Wang Y, Yisimayi A, Wang W, Zhang W, Du J, Yu H, Xie XS, Jin R. Humoral immunogenicity and reactogenicity of CoronaVac or ZF2001 booster after two doses of inactivated vaccine. Cell Res 2021; [Epub ahead of print] doi: 10.1038/s41422-021-00596-5

19. Zeng G, Wu Q, Pan H, Li M, Yang J, Wang L, Wu Z, Jiang D, Deng X, Chu K, Zheng W, Wang L, Lu W, Han B, Zhao Y, Zhu F, Yu H, Yin W. Immunogenicity and safety of a third dose of CoronaVac, and immune persistence of a two-dose schedule, in healthy adults: interim results from two single-centre, double-blind, randomised, placebo-controlled phase 2 clinical trials. Lancet Infect Dis 2021; [Epub ahead of print] doi: 10.1016/S1473-3099(21)00681-2

20. Bar-On YM, Goldberg Y, Mandel M, Bodenheimer O, Freedman L, Kalkstein N, Mizrahi B, Alroy-Preis S, Ash N, Milo R, Huppert A. Protection of BNT162b2 vaccine booster against Covid-19 in Israel. N Engl J Med 2021; 385(15): 1393-1400

21. Falsey AR, Frenck RW Jr, Walsh EE, Kitchin N, Absalon J, Gurtman A, Lockhart S, Bailey R, Swanson KA, Xu X, Koury K, Kalina W, Cooper D, Zou J, Xie X, Xia H, Türeci Ö, Lagkadinou E, Tompkins KR, Shi PY, Jansen KU, Şahin U, Dormitzer PR, Gruber WC. SARS-CoV-2 neutralization with BNT162b2 vaccine dose 3. N Engl J Med 2021; 385(17): 1627-1629

22. Hillus D, Schwarz T, Tober-Lau P, Vanshylla K, Hastor H, Thibeault C, Jentzsch S, Helbig ET, Lippert LJ, Tscheak P, Schmidt ML, Riege J, Solarek A, von Kalle C, Dang-Heine C, Gruell H, Kopankiewicz P, Suttorp N, Drosten C, Bias H, Seybold J; EICOV/ COVIM Study Group, Klein F, Kurth F, Corman VM, Sander LE. Safety, reactogenicity, and immunogenicity of homologous and heterologous prime-boost immunisation with ChAdOx1 nCoV-19 and BNT162b2: a prospective cohort study. Lancet Respir Med 2021; 9(11): 1255-1265

23. Munro APS, Janani L, Cornelius V, Aley PK, Babbage G, Baxter D, Bula M, Cathie K, Chatterjee K, Dodd K, Enever Y, Gokani K, Goodman AL, Green CA, Harndahl L, Haughney J, Hicks A, van der Klaauw AA, Kwok J, Lambe T, Libri V, Llewelyn MJ, McGregor AC, Minassian AM, Moore P, Mughal M, Mujadidi YF, Murira J, Osanlou O, Osanlou R, Owens DR, Pacurar M, Palfreeman A, Pan D, Rampling T, Regan K, Saich S, Salkeld J, Saralaya D, Sharma S, Sheridan R, Sturdy A, Thomson EC, Todd S, Twelves C, Read RC, Charlton S, Hallis B, Ramsay M, Andrews N, Nguyen-Van-Tam JS, Snape MD, Liu X, Faust SN; COV-BOOST study group. Safety and immunogenicity of seven COVID-19 vaccines as a third dose (booster) following two doses of ChAdOx1 nCov-19 or BNT162b2 in the UK (COV-BOOST): a blinded, multicentre, randomised, controlled, phase 2 trial. Lancet 2021; 398 (10318): 2258-2276 